\title{
MECHANICAL PROPERTIES AND FLUORIDE RELEASE OF GLASS IONOMER CEMENT WITH ENHANCED ANTIMICROBIAL ACTIVITY DERIVED FROM ADDITION OF SCHIFF BASE OF TETRONIC 1107
}

\author{
Samy M El-Safty*, El-Refaie S Kenway**, Mohamed M. Azaam***, \\ Yehia A Mahmoud**** and Samar A Khattab*****
}

\begin{abstract}
Objectives: To synthesize and characterize the Schiff base of Tetronic 1107 (T1107SB) to be added to a well-known GIC to enhance its antimicrobial activities and to evaluate the compressive strength (CS), elastic modulus (E), Vickers microhardness (HV) and fluoride release (FR) of the synthesized GIC.
\end{abstract}

Materials and methods: T1107SB was synthesized by the chloroacetylation of T1107, amination of chloroacetylated T1107 and modification of aminated T1107 with vanillin. Characterization of T1107SB was carried out by Fourier transform infra-red spectroscopy (FTIR), Elemental microanalysis and Thermal gravimetric analysis (TGA). The antimicrobial activity of T1107SB-modified GIC (T-GIC) was studied by the "cut plug method" and minimal inhibitory concentration (MIC) test. CS and $E$ were investigated in a universal testing machine according to the ISO 9917:2007. HV was evaluated by a Digital Microhardness Tester (Zwick/Roell, IDENTEC, ZHV $\mu$-S, West Midlands, England). Fluoride levels in ppm were obtained using the ion-selective electrode connected to a digital meter. A one-way ANOVA and Bonferroni test were used to analyze the data with the significance level established at $(\mathrm{p} \leq 0.05)$.

Results: Synthesis of T1107SB was confirmed by FTIR, elemental micro-analysis and TGA. T-GICs exhibited an enhanced antibacterial activity, compared to the control GIC (C-GIC) against Staphylococcus aureus and Bacillus cereus as confirmed by the growth inhibition zones. T-GICs showed no improvement in the antimicrobial activity against Candida albicans, compared to C-GIC. Significant differences were found between studied groups in CS, E, HV and FR. There was a systematic reduction in such properties with increasing the added percent of T1107SB.

\footnotetext{
* Lecturer, Department of Dental Biomaterials, Faculty of Dentistry, Tanta University, Tanta, Egypt.

** Professor of Polymer Chemistry, Department of Chemistry, Polymer Research Group, Faculty of Science, Tanta University, Tanta, Egypt.

*** Assistant Professor of Polymer Chemistry, Department of Chemistry, Polymer Research Group, Faculty of Science, Tanta University, Tanta, Egypt.

**** Professor of Microbiology (Mycology), Faculty of Science, Tanta University, Tanta, Egypt

***** Assistant Lecturer, Department of Chemistry, Faculty of Science, Tanta University, Tanta, Egypt.
} 
Conclusions: Addition of T1107SB to GIC powder is promising in enhancing the antibacterial activity of GIC materials. Despite the reduction in CS, E, HV and FR because of the addition of T1107SB, such modified T-GICs can be used with a guaranteed degree of clinical success.

Keywords: Antimicrobial Activity; Tetronic 1107; Schiff Base; Compressive Strength; Elastic Modulus; Microhardness; Fluoride Release.

\section{INTRODUCTION}

Glass ionomer cements (GICs) have been applied in daily dental practice for more than five decades. The chemical bonding to the tooth structure, fluoride release, low coefficient of thermal expansion/contraction, adequate esthetic properties and biocompatibility with the pulp tissues are the main encouraging characteristics of their dental uses ${ }^{[1,2]}$. Because of suffering from some shortcomings, particularly the mechanical properties, GICs have been modified and improved. Addition of resin materials to produce resin-modified GICs (RMGICs) represents one major modification of these materials. Such a modification produced a GIC with enhanced mechanical properties and lower solubility while maintaining the fluoride release and anti-carious potential ${ }^{[3-5]}$.

Another important improvement of GICs was accomplished by the incorporation of antimicrobial agents ${ }^{[6,7]}$. This rendered GICs able to prevent the occurrence of secondary caries by inhibiting growth of invading bacteria. Addition of chlorhexidine (CHX), in the form of chlorhexidine acetate, chlorhexidine gluconate or chlorhexidine hydrochloride, to GICs was accepted by many authors because of its antibacterial activities against cariogenic bacteria ${ }^{[8]}$. Incorporation of $\mathrm{CHX}$ into GICs, though affecting physical and mechanical properties and bonding capacity, was said to have a positive impact on fluoride release ${ }^{[9]}$. In the same stream, a variety of antibacterial polymers were added to GICs. Epigallocatechin-3-gallate (EGCG) was reported to have an antimicrobial function to control oral streptococci and can act as a promising cariostatic agent ${ }^{[10]}$. Polymers that contain quaternary ammonium salt (QAS) or quaternary phosphonium salt (QPS) were reported to have antimicrobial activities and could be useful to enhance the restorative materials to overcome secondary caries ${ }^{[11]}$. Furanone-modified GICs were also introduced and proved to have long-term antibacterial activities [12]. Addition of propolis to GICs was also studied and results revealed an antibacterial activity against cariogenic bacteria particularly Streptococcus mutans and sobrinus ${ }^{[13]}$. Chitosan-modified GIC was also introduced and evaluated.

Chitosan $(\mathrm{CH})$ is a linear bio-polyaminosaccharide derived from alkaline deacetylation of chitin. Addition of $10 \%$ (by volume) of $\mathrm{CH}$ reported an enhancement in flexural strength and fluoride release of GIC, in addition to the antibacterial activity ${ }^{[14]}$.

An important class of the most widely used organic compounds is "Schiff bases". These compounds have been shown to exhibit a variety of biological activities such as antibacterial, antifungal, antiviral, anti-inflammatory, antimalarial and antipyretic properties ${ }^{[15,16]}$. Schiff bases, firstly studied by Hugo Schiff 1864, are formed by the reaction of any primary amine with an aldehyde or a ketone under specific conditions. Structurally, a Schiff base is a nitrogen analogue of an aldehyde or ketone in which the carbonyl group $(\mathrm{C}=\mathrm{O})$ has been replaced by an imine or azomethine group ${ }^{[17]}$. It was found that the imine group present in such compounds is very critical to their biological activities ${ }^{[18,19]}$. 
Tetronics, a category of Schiff bases, are $\mathrm{X}$-shaped polyethers with four poly(ethylene oxide-propylene oxide) (PEO-PPO) arms bonded to a central ethylene diamine linker, as seen in Figure 1. They are widely used in many applications such as antifoaming agents, wetting agents, dispersants, thickeners, and emulsifiers for different industrial purposes ${ }^{[20]}$. Recently, they were applied in biomedical and pharmaceutical fields such as drug delivery, genetic immunization, and membrane biochemistry ${ }^{[21,22]}$. The antibacterial activity of these compounds, particularly T1107, was evaluated and the study concluded a significant inhibiting effect on all tested microorganisms ${ }^{[23]}$.

The current study aimed to synthesize and characterize the Schiff base of Tetronic 1107 (T1107SB) and to add this compound to a wellknown GIC to produce a modified GIC with enhanced antimicrobial activities. While preparing such a modified GIC, it is very essential to maintain adequate mechanical properties such as acceptable strength, modulus and microhardness. At the same time, it is very essential that fluoride ion-releasing capacity should not be affected by the addition of the synthesized antimicrobial agent. Therefore, the main objectives of this study were: synthesis and characterization of T1107SB, addition of this product to a well-marketed GIC to prepare a GIC with enhanced antimicrobial activity and evaluation of the compressive strength, elastic modulus,
Vickers microhardness and fluoride ion release of the modified GIC. The null hypotheses of the study were: i) there will be no difference between the antimicrobial activity of control GIC and that of modified GICs and ii) addition of T1107SB to the GICs will have no effect on compressive strength, elastic modulus, Vickers microhardness and fluoride ion release.

\section{MATERIALS AND METHODS}

\section{Materials}

A commercially available glass ionomer cement (GIC) Fuji IX (glass powder and polyacrylic acid) (GC International, Tokyo, Japan) was used. Tetronic 1107 (T1107) (Average molecular weight: $15000 \mathrm{~g} /$ mol) was obtained from BASF Corporation (New Jersey, USA). Chloroacetyl chloride and Glacial acetic acid were purchased from El-Gomhouria Chemicals Company (Cairo, Egypt). Pyridine was purchased from El-Nasr Pharmaceutical Chemicals (Cairo, Egypt). p-Phenylene diamine was purchased from Acros Organics (Belgium). Vanillin was purchased from Sigma-Aldrich Chemical Co. (Milwaukee, WI, USA). Diethyl ether was purchased from El-Nasr Pharmaceutical Chemicals Company (Cairo, Egypt) and dried using a molecular sieve with a pore size of 4 angstrom (4A) before use. Absolute ethanol was purchased from Merck-Schuchardt, Hohenbrunn (Germany).

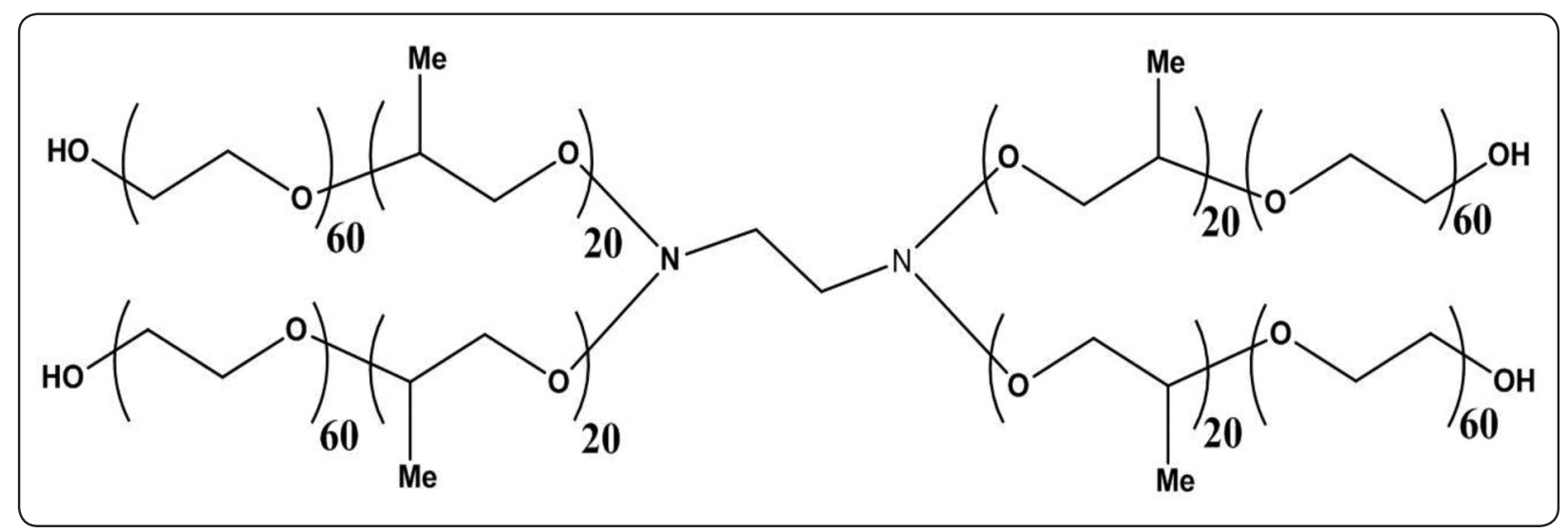

Fig. (1) Chemical structure of Tetronic 1107 


\section{Methods:}

\section{Synthesis of Schiff base of T1107:}

\section{Chloroacetylation of T1107 (II)}

In a round flask ( $100 \mathrm{ml}), 19 \mathrm{ml}$ of Pyridine (240 mmol) was added to a solution of T1107 (10 g, 20 $\mathrm{mmol}$ ) in dry ethanol and the mixture was cooled to $0{ }^{\circ} \mathrm{C}$. Chloroacetyl chloride $(19 \mathrm{ml}, 240 \mathrm{mmol})$ was added to the cold mixture dropwise with vigorous stirring (DAIHAN MaXtir ${ }^{\mathrm{TM}}$ 500S Hi-performance Digital Magnetic Stirrers, SRICO, South Korea). The reaction mixture was stirred at $0^{\circ} \mathrm{C}$ for $3 \mathrm{~h}$ and at room temperature for further $48 \mathrm{~h}$. The excess ethanol was removed by a rotary evaporator (IKA RV 10 V Digital Rotary Evaporator ,115 VAC, Germany). The precipitate was washed with diethyl ether and the product was dried under vacuum (Model VD 53, BINDER, Germany) for $10 \mathrm{~h}$.

\section{Amination of chloroacetylated T1107 (III)}

To a solution of $p$-phenylenediamine (13 g, 120 $\mathrm{mmol}$ ) in dry ethanol, chloroacetylated T1107 (5 g, $6 \mathrm{mmol}$ ) were added portionwise. After the addition was completed, the system was fitted to reflux at 80 ${ }^{\circ} \mathrm{C}$ for 4 days with continuous stirring. The product was washed with ethanol to remove the unreacted diamine. The product was then dried under vacuum for $48 \mathrm{~h}$.

\section{Formation of Schiff base from amine-modified T1107 and vanillin) (IV)}

To a solution of vanillin $(1.08 \mathrm{~g}, 7.13 \mathrm{mmol})$ in $15 \mathrm{ml}$ of absolute methanol, aminated T1107 (1g, $0.89 \mathrm{mmol}$ ) and $1 \mathrm{ml}$ of glacial acetic acid were added with stirring. Stirring was continued at room temperature for $48 \mathrm{~h}$ and then the system was fitted to reflux at $80{ }^{\circ} \mathrm{C}$ for $10 \mathrm{~h}$. The formed precipitate was filtered off and washed with methanol to remove the excess vanillin and acetic acid. The product was collected as powder and dried under vacuum at room temperature for $48 \mathrm{~h}$.

\section{Characterization of T1107 and its Schiff base:}

\section{Fourier transform infra-red spectroscopy (FTIR)}

FTIR spectra were obtained using a ratio recording spectrophotometer (Perkin-Elmer, model 1430) over the wavenumber range $4000-400 \mathrm{~cm}^{-1}$. Samples from T1107 and T1107SB were prepared as $\mathrm{KBr}$ pellets by mixing $1 \mathrm{mg}$ of each with $200 \mathrm{mg}$ $\mathrm{KBr}$ powder. After thorough mixing with $\mathrm{KBr}$, the mix was pulverized, put into a pellet-forming die and pressed under a hydraulic press to form a tablet. The samples were scanned against a blank pellet background of $\mathrm{KBr}$ within the wavelength range of $4000-4000 \mathrm{~cm}^{-1}$ with a resolution of $4 \mathrm{~cm}^{-1}$.

\section{Elemental micro-analysis}

This investigation was conducted using Elemental Analyzer Model 1106, Carlo Erba Strumentazione, (Milan, Italy). A sample from T1107 and its derivatives (II, III and IV) with a mass of no more than $1 \mathrm{mg}$ was burned in a vertical reactor (oxidation tube) in the dynamic mode at a temperature of about $1020^{\circ} \mathrm{C}$ in a helium (He) flow with the addition of $\mathrm{O}_{2}(10 \mathrm{ml})$ at the instant of sample introduction. The elemental analyzer software calculates the amounts of the resultant gases as a percentage of the initial sample weight. The results show $\% \mathrm{C}, \% \mathrm{H}, \% \mathrm{~N}$. In addition, these percents were calculated (mathematically) and the measured values were compared to those calculated. Calculations were carried out as follows: The mass of the repeating unit of a polymer could be calculated by looking up the atomic masses of each of the elements and adding them together. If an element has a subscript following its symbol, the mass of that element is multiplied by the subscript before doing the summation. Once we know the mass of the compound, the theoretical percent of each element could be calculated by dividing the atomic mass of that element - multiplied by the subscript that follows it in the formula - by the mass of the compound and then multiplying by 100 . 


\section{Thermal gravimetric analysis (TGA)}

This analysis was carried out using a thermogravimetric analyzer (Perkin-Elmer TGA 4000, USA). This analysis determines the thermal stability (degradation) of T1107 and the synthesized Schiff base. Samples of T1107 and its Schiff base (2 $\mathrm{mg}$ ) were gradually heated over a temperature range of $50-800{ }^{\circ} \mathrm{C}$ and at a scanning rate of $20^{\circ} \mathrm{C} \mathrm{min}^{-1}$ under a nitrogen atmosphere with a flow rate of 20 $\mathrm{ml} / \mathrm{min}$. As a derivative of TGA, a test known as derivative thermogravimetry (DTG) was conducted as well. DTG is a type of thermal analysis in which the rate of a material's weight changes upon heating is plotted against temperature and used to simplify reading the weight versus temperature. DTG indicates the major degradation regions and tells us at what temperature major degradation occurs.

\section{Formulation and grouping of T1107SB-modified GICs}

To prepare the experimental GIC groups (T1107-modified GICs), part of the GIC powder was replaced with a corresponding weight of T1107SB powder and thoroughly hand-mixed until a homogenous mixture of the two powders was obtained. The powder of T1107SB was added to GIC powder at the percents of $2 \%, 4 \%, 6 \%$ and $10 \%$ to form four modified (T-GIC) groups, in addition to the control (C-GIC) group as follows:

C-GIC: GIC without T1107SB.

T-GIC-2: 98 wt $\%$ of GIC mixed with 2 wt $\%$ T1107SB.

T-GIC-4: 96 wt\% of GIC mixed with 4 wt $\%$ T1107SB.

T-GIC-6: 94 wt $\%$ of GIC mixed with 6 wt $\%$ T1107SB.

T-GIC-10: 90 wt $\%$ of GIC mixed with $10 \mathrm{wt} \%$ T1107SB.

\section{Antimicrobial activity}

\section{Tested microorganisms}

The antimicrobial activity of T-GICs and C-GIC was evaluated against the Gram-positive bacteria; Staphylococcus aureus and Bacillus cereus and Candida albicans SC5314. The microorganisms were obtained from Microbiology section, Botany Department, Faculty of Science. The bacterial strains were maintained and assayed on nutrient agar which contained $3 \mathrm{~g}$ peptone, $5 \mathrm{~g} \mathrm{NaCl}, 5 \mathrm{~g}$ beef extract, and $20 \mathrm{~g}$ agar per litre.

\section{Screening of antimicrobial activity}

The antimicrobial activity of the tested samples was determined using "the cut plug method" [24], where the combined T1107SB-GICs were not soluble in water or any other solvent. A $0.5 \mathrm{ml}$ of microorganism $\left(10^{7} \mathrm{CFU} / \mathrm{ml}\right)$ suspension of cells was prepared and accounted and then mixed with 9.5 $\mathrm{ml}$ of the sterilized melted nutrient agar and left to solidify at room temperature. Powder test samples (100 mg) of both T-GICs and C-GIC were weighed. Wells were created in the agar plates and the powder was mixed with the liquid according to the manufacturer's instructions and then applied into each well to detect the most sensitive microorganisms. The plates were incubated at $37^{\circ} \mathrm{C}$ for $24 \mathrm{~h}$ then the zones of bacterial growth inhibition were recorded in millimeters (mm) using a digital caliper (Mitutoyo, Tokyo, Japan). Measurements were taken at the greatest distance between two points at the outer border of the inhibition halo formed around the well. This measurement was repeated three times and the mean was computed for each well.

\section{Minimal inhibitory concentrations (MICs)}

In this investigation, the minimal inhibitory concentration (MIC) value was tested against $\mathrm{S}$. aureus and B. cereus by measuring the Organisms Surviving Ratio (OSR) for both organisms. A suspension of each standard organism $\left(0.5 \mathrm{ml} ; 10^{7}\right.$ $\mathrm{CFU} / \mathrm{ml}$ ) was mixed with $9.5 \mathrm{ml}$ of the 10 -fold 
diluted corresponding media broth in a sterile test tube. The amount of T1107SB:GIC was added to give different concentrations as $\mu \mathrm{g} / \mathrm{ml}$ for group $V$ (10 T1107SB:90 GIC) in nutrient broth in the final solution. The seeded tubes were then shaken at 250 $\mathrm{rpm}$ at $37^{\circ} \mathrm{C}$ overnight. The solutions were then diluted 3-fold and $100 \mu 1$ of each solution was spread onto agar plates of corresponding media. Plates from $\mathrm{C}$-GIC were prepared and incubated at $37^{\circ} \mathrm{C}$ for 24 $\mathrm{h}$, and then the number of colonies forming units (CFU) was recorded as well. The surviving ratio $(\mathrm{M} / \mathrm{C})$ was calculated for each chosen organism at different T-GIC concentrations against that of the control, where $\mathrm{M}$ is the number of organisms in presence of different T-GIC concentrations; $\mathrm{C}$ is the number of organisms in absence of T1107SB (control).

\section{Testing of mechanical properties of T1107-mod- ified GICs}

\section{Compressive strength (CS) and elastic modulus $(E)$}

The cement powders were mixed with the liquid at the ratio of $(\mathrm{P} / \mathrm{L}=3.6 \mathrm{~g} / \mathrm{g})$ as recommended by the manufacturer. Cylindrical specimens $(n=10)$ were prepared in Teflon molds with the dimensions of $4 \mathrm{~mm}$ in diameter and $6 \mathrm{~mm}$ in height. Within $60 \mathrm{~s}$ after the end of mixing, the mixed cement was packed to the molds with a slight overfilling and then gently compressed between two glass plates covered with transparent polystyrene matrix films. One hour after the end of mixing, specimens were removed from the molds and stored in distilled water at $37^{\circ} \mathrm{C}$ in an incubator for $24 \mathrm{~h}$. Just before testing, specimens of each group were slightly dried with a sheet of damp filter paper (Whatman No. 1) and a compressive load was applied along the long axis of the specimen, at a cross-head speed of 0.75 $\mathrm{mm} / \mathrm{min}$ using a universal testing machine (Zwick/ Roell Z020, Leominster, UK). Prior to examination, each specimen was checked for accurate dimensions using a digital caliper (Mitutoyo, Tokyo, Japan). The maximum load used to fracture the specimen was divided by the cross-section area to obtain the value of the compressive strength $\left(\boldsymbol{\sigma}_{c}\right)$ for each specimen in MPa as indicated in ISO 9917:2007 ${ }^{[25]}$ according to the following formula:

$$
\sigma_{c}=\frac{F}{A}
$$

where $F$ is the fracture load $(\mathrm{N})$ and $A$ is the area $\left(\mathrm{mm}^{2}\right)$ of the cylindrical specimen.

The elastic modulus $(E)$ was calculated according to the following formula ${ }^{[26]}: E=\Delta \delta / \Delta \varepsilon$, where $\Delta \delta$ (stress) equals $\Delta \mathrm{F}_{1} / \mathrm{A}$, where $F_{1}$ is the load $(\mathrm{N})$ applied at the point 1 of the straight portion of the trace, $A$ is the cross-sectional area of the test specimen $\left(\mathrm{mm}^{2}\right)$, $\Delta \varepsilon$ (strain) is $\Delta l / L$, where $L$ is the specimen height and $l_{i}$ is the deflection corresponding to load $F_{l}$ at a point in the straight portion of the stress-strain curve.

\section{Vickers microhardness (HV)}

A total of 10 disc-shaped specimens $(8 \mathrm{~mm}$ diameter $\times 2 \mathrm{~mm}$ thickness) were prepared in Teflon molds. Glass microscope slides, covered with transparent polystyrene matrix films, were positioned at the upper and lower surfaces of the specimen and pressed under hand pressure to extrude excess material. After complete setting, excess material around the mold was removed by hand-grinding with 800 grit silicon carbide paper. Specimens were then removed from the mold and stored in distilled water for $24 \mathrm{~h}$ before testing. Hardness evaluation was conducted using a Digital Microhardness Tester (Zwick/Roell, IDENTEC, $\mathrm{ZHV} \mu-\mathrm{S}$, West Midlands, England) by applying a load of $2.9 \mathrm{~N}$ force for $15 \mathrm{~s}^{[27,28]}$. Each specimen was fixed in a clamping apparatus and positioned in a manner that the indenter tip will be perpendicular to the specimen surface to be tested. All results were generated and reported in HV units by using the microhardness tester. The Vickers hardness number (VHN) $\left(\mathrm{kgf} / \mathrm{mm}^{2}\right)$ for each tested specimen was measured according to the following equation ${ }^{[25]}$ :

$$
\mathrm{VHN}=1.8544 \times \mathrm{L} / d^{2}
$$

where $L$ is the applied load (kgf) and $d$ is mean diagonal length $(\mathrm{mm})$. 
Each specimen was subjected to five indentations equally-spaced over a circle. Care was taken to make the indentation not closer than $1 \mathrm{~mm}$ to the adjacent indentations or the margin of the specimen. The average of the five indentations per specimen was then calculated and the mean of ten specimens was taken as the microhardness of the group.

\section{Fluoride-release measurement}

A total of ten disc-shaped specimens $(10 \mathrm{~mm}$ in diameter and $2 \mathrm{~mm}$ in thickness) from each GIC group were prepared in Teflon molds. After complete setting, each specimen was immersed in $20 \mathrm{ml}$ of de-ionized water in a closed plastic container at 37 ${ }^{\circ} \mathrm{C}$ for $24 \mathrm{~h}$. Measurement of fluoride ion was carried out by removing the specimen from its container and the storage solution was collected for analysis. Specimens were gently dried and then placed in a new container with fresh $20 \mathrm{ml}$ of de-ionized water. The ion-selective electrode method ${ }^{[28,29]}$ was applied for measuring the fluoride ion concentration at $24 \mathrm{~h}, 48 \mathrm{~h}, 1$ week and 1 month. Results were calculated as the amount of fluoride per unit surface area of the specimen $\left(\mu \mathrm{g} / \mathrm{mm}^{2}\right)$. Fluoride levels in ppm were obtained using the ion-selective electrode connected to a digital meter. The total fluoride ion in $\mu \mathrm{g}$ was calculated by multiplying the $1 \mathrm{ppm}(=1 \mu \mathrm{g} /$ $\mathrm{ml})$ by the tested solution volume $(20 \mathrm{ml})$. After that, the total fluoride was then divided by the area of the tested specimen to obtain the fluoride release in $\mu \mathrm{g} / \mathrm{mm}^{2}$.

\section{Statistical analysis}

Data analysis was carried out using an IBM compatible personal computer with SPSS statistical package version 20 (SPSS Inc. Released 2011. Armnok, NY: IBM Corp.). A one-way analysis of variance (ANOVA) with the significance level established at ( $\mathrm{p} \leq 0.05)$ was applied for analyzing the results of studied properties. As significant differences were found between groups of compressive strength, elastic modulus and hardness, Levene's test for homogeneity of variances was carried out for the data of each property $(\mathrm{p} \leq$ $0.05)$ to choose the appropriate test for multiple comparisons. Equal variances were confirmed ( $p>$ $0.05)$; therefore, the Bonferroni test was applied.

\section{RESULTS}

\section{Synthesis of T1107SB:}

Figure 2 shows the chloroacetylation of T1107 (II), amination of chloroacetylated T1107 (III) and modification of aminated T1107 with vanillin to form T1107 Schiff base (IV).

\section{Characterization results of T1107SB:}

\section{Fourier transform infra-red spectroscopy (FTIR)}

Figure 3 shows the FTIR of T1107 (I), chloroacetylated T1107 (II), amination of chloroacetylated T1107 (III) and Schiff base of T1107 (IV). The FTIR spectrum of T1107 is characterized by principal absorption peaks at $3446 \mathrm{~cm}^{-1}$ (O-H stretching vibrations), 2904 $\mathrm{cm}^{-1}$ (C-H stretching vibrations), and $1109 \mathrm{~cm}^{-1}$ (C-O-C stretching vibrations). All characteristic absorption bands of T1107 were also observed on the chloroacetylated T1107 spectrum (II) that showed presence of new peaks at $753 \mathrm{~cm}^{-1}$ assigned for stretching $\left(-\mathrm{CH}_{2} \mathrm{Cl}\right)$ and $1740 \mathrm{~cm}^{-1}$ assigned for carbonyl group stretching $(-\mathrm{C}=\mathrm{O})$ which confirms the chloroacetylation of terminal hydroxyl groups of T1107. The FTIR spectrum of aminated T1107 (III) showed peaks at 3399 and $3321 \mathrm{~cm}^{-1}$ attributed to the new amino group $\left(\mathrm{NH}_{2}\right)$, strong band at 829 $\mathrm{cm}^{-1}$ for C-H (aromatic) and peaks at 1510 and 1571 $\mathrm{cm}^{-1}$ for $\mathrm{C}=\mathrm{C}$ (aromatic). The FT-IR spectrum of T1107 Schiff base (IV) showed the disappearance of the primary amino group. Instead, a strong peak appeared at $1596 \mathrm{~cm}^{-1}$ which belongs to the azomethine group $(\mathrm{C}=\mathrm{N})$ due to the reaction of aminated T1107 with vanillin that confirms the formation of the Schiff base. Also, there were peaks at $2963 \mathrm{~cm}^{-1}$ for $\mathrm{C}-\mathrm{H}$ (aliphatic), at $3211 \mathrm{~cm}^{-1}$ for $\mathrm{N}-\mathrm{H}$ and at $823 \mathrm{~cm}^{-1}$ for C-H (aromatic). 


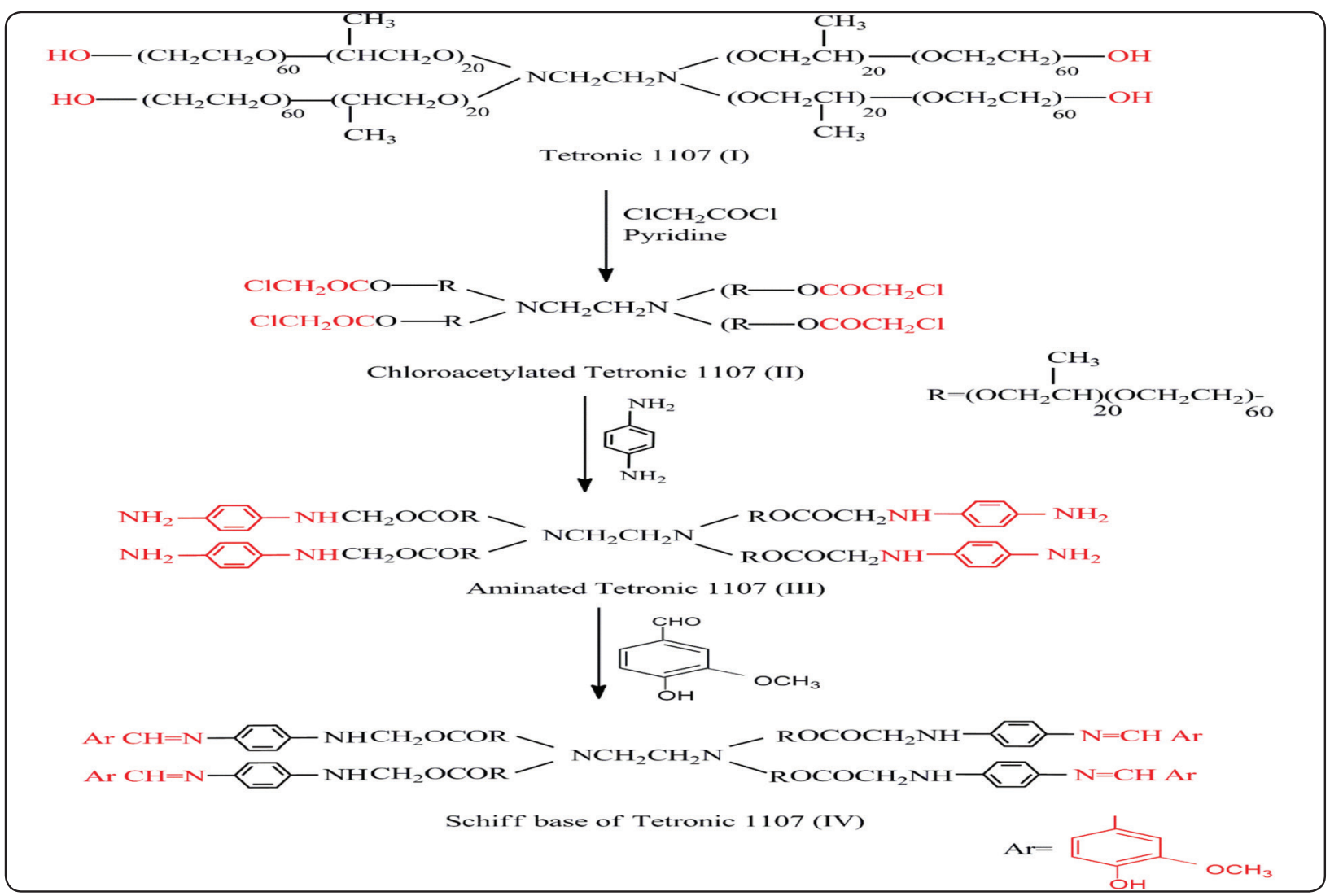

Fig. (2) Synthesis of Schiff base of Tetronic 1107

\section{Elemental micro-analysis}

Percents of $\mathrm{C}, \mathrm{H}$ and $\mathrm{N}$ of $\mathrm{T} 1107$ and its derivatives (I-VI) measured by the elemental microanalyzer and those calculated are listed in Table 1. Calculation of $\%$ of C, H and $\mathrm{N}$ of T1107 (I-IV) was based on their given chemical formulas as follows: T1107 (I) $=\mathrm{C}_{22} \mathrm{H}_{48} \mathrm{~N}_{2} \mathrm{O}_{12}$; molecular weight $=532 \mathrm{~g} /$ mole, chloroacetylated T1107 (II): $\mathrm{C}_{30} \mathrm{H}_{52} \mathrm{~N}_{2} \mathrm{O}_{16} \mathrm{Cl}_{4}$; molecular weight $=838 \mathrm{~g} /$ mole, aminated $\mathrm{T} 1107$ (III): $\mathrm{C}_{54} \mathrm{H}_{80} \mathrm{~N}_{10} \mathrm{O}_{16}$; molecular weight $=1124 \mathrm{~g} /$ mole and T1107SB (IV): $\mathrm{C}_{86} \mathrm{H}_{104} \mathrm{~N}_{10} \mathrm{O}_{24}$; molecular weight $=1660 \mathrm{~g} / \mathrm{mole}$.

\section{Thermal gravimetric analysis (TGA)}

Figure 4 shows the thermal degradation pattern of T1107 and its Schiff base produced by TGA. The TGA patterns of T1107 showed a weight loss at a temperature range from 224 to $430{ }^{\circ} \mathrm{C}$ with a singlestage decomposition. T1107 Schiff base, on the other hand, showed a multi-stage decomposition. The first stage begins at a range from $265^{\circ} \mathrm{C}$ to $487^{\circ} \mathrm{C}$. The first region of weight loss, up to $152{ }^{\circ} \mathrm{C}$, reflects removal of solvent and moisture. The onset thermal degradation (defined as 8.4 wt\% loss) of T1107 Schiff base was observed at $258{ }^{\circ} \mathrm{C}$ which was $145^{\circ} \mathrm{C}$ lower than unmodified T1107 $\left(403.2^{\circ} \mathrm{C}\right)$. A weight loss of 50 wt $\%$ of both T1107 and T1107 Schiff base were observed at $381{ }^{\circ} \mathrm{C}$ and $504{ }^{\circ} \mathrm{C}$, respectively. The temperature of maximum rate of weight loss (largest decomposition step) could be determined from the first derivative of the TGA curve (known as derivative thermogravimetry "DTG") which was $403.2^{\circ} \mathrm{C}$ for unmodified $\mathrm{T} 1107$ and $258.4^{\circ} \mathrm{C}$ for T1107SB, as can be shown in Figure 5. 


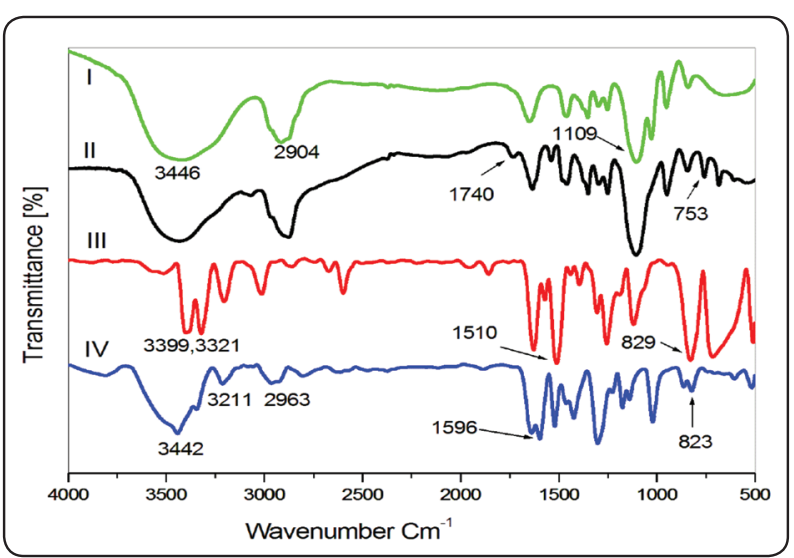

Fig. (3) FTIR spectra of T1107 (I), chloroacetylated T1107 (II), aminated T1107 (III) and T1107SB (IV).

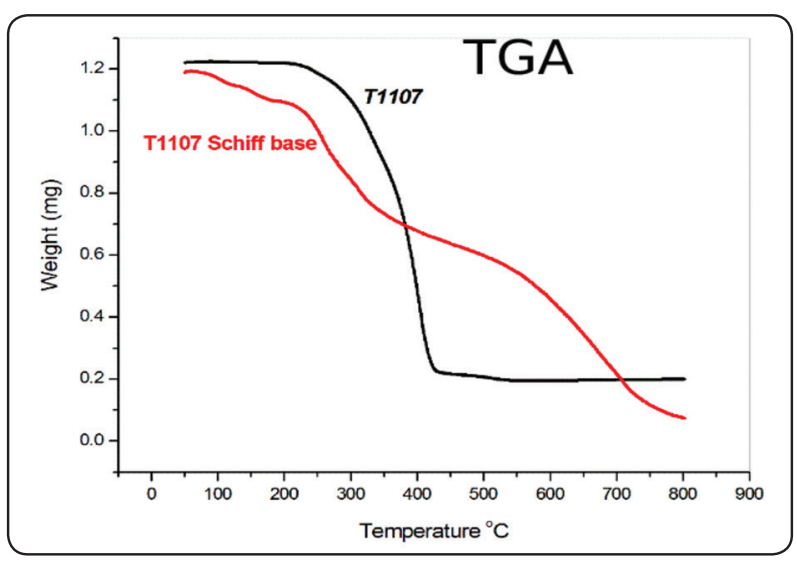

Fig. (4) Curves of thermal gravimetric analysis of T1107 (black) and T1107 Schiff base (red).

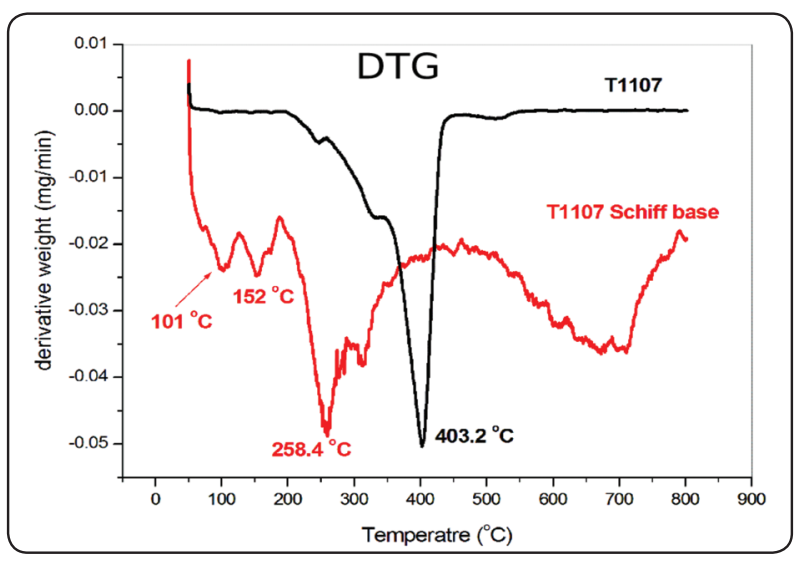

Fig. (5) DTG Curves of derivative thermogravimetry of T1107 and T1107 Schiff base.

\section{Antimicrobial activity:}

The inhibition zones (in $\mathrm{mm}$ ) of C-GIC and T-GICs against the tested microorganisms ( $S$. aureus, B. cereus and C. albicans) are listed in Table 2. Figure 6 presents the inhibitory effect of T-GICs compared to C-GIC in case of S. aureus and B. cereus. In case of $S$. aureus and B. cereus, the first modified group (GIC 98\%:T1107 2\%) recorded no effect on the growth inhibition zone compared to the control group. Other T-GICs demonstrated greater inhibition zones than C-GIC. There was a systematic increase in the inhibition zone recorded for the T-GICs with increasing the T1107 SB percent at the expense of GIC. For C. albicans, however, addition of T1107 (within the range selected, 2-10\%) to GIC revealed no difference in the inhibition zones compared to the control group. Higher sensitivity was exhibited by B. cereus compared to $S$. aureus both in C-GIC and T-GICs.

TABLE (2) Diameter of inhibition zones ( $\mathrm{mm}$ ) of C-GIC and T-GICs against tested microorganisms.

\begin{tabular}{|c|c|c|c|}
\hline $\begin{array}{l}\text { Mroups } \\
\text { (T1107 SB \%) }\end{array}$ & S. aureus & B.cereus & C.albicans \\
\hline Group I: (0 \%) & $15 \pm 1.0$ & $24 \pm 1.0$ & $13 \pm 0.6$ \\
\hline Group II: (2 \%) & $15 \pm 1.0$ & $24 \pm 2.0$ & $13 \pm 0.6$ \\
\hline Group III: (4\%) & $17 \pm 0.6$ & $25 \pm 1.0$ & $13 \pm 0.7$ \\
\hline Group IV: (6 \%) & $18 \pm 0.8$ & $26 \pm 2.7$ & $13 \pm 0.6$ \\
\hline Group V: (10 \%) & $19 \pm 0.4$ & $30 \pm 1.2$ & $13 \pm 0.8$ \\
\hline
\end{tabular}

The minimal inhibitory concentration (MIC) test for group V of T-GICs against $S$. aurues revealed $500 \pm 5.0 \mu \mathrm{g} / \mathrm{ml}$ and $250 \pm 3.0 \mu \mathrm{g} / \mathrm{ml}$ in case of $B$. cereus (Table 3 ). 


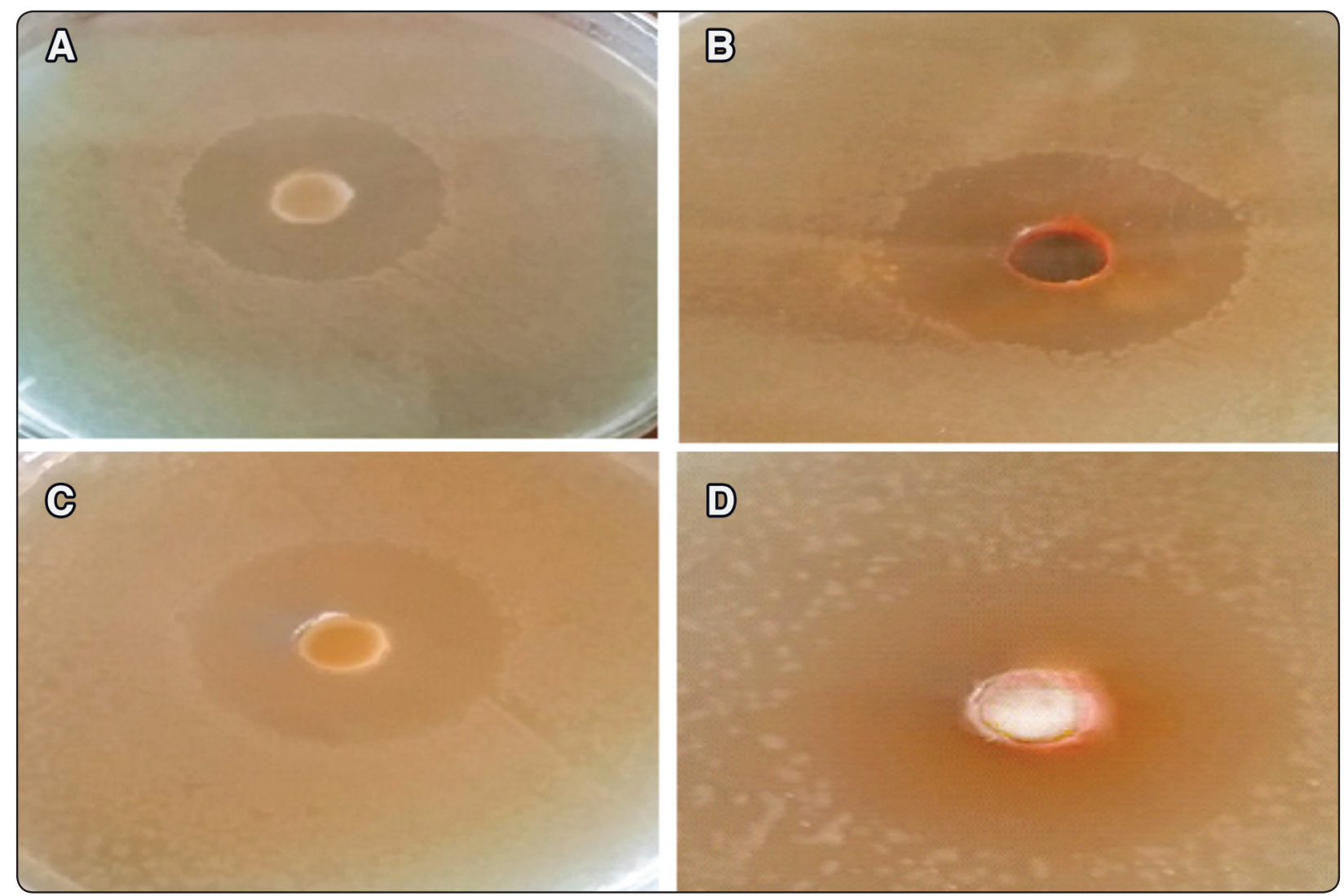

Fig. (6) Inhibition zones of: (A) C-GIC and (B) T-GIC against S. aureus and (C) C-GIC and (D) T-GIC against B. cereus. Group III of T-GICs (4\% T1107 SB and 96\% GIC) was used as a representative of the modified groups.

TABLE (3) Minimal inhibitory concentration (MIC) of group V T-GICs against $B$. cereus and S. aureus.

\begin{tabular}{|c|c|c|}
\hline T-GICs & Microorganism & MIC $(\boldsymbol{\mu g} / \mathbf{m l})$ \\
\hline Group $V$ & S. aureus & $500 \pm 5.5$ \\
\hline Group $V$ & B. cereus & $250 \pm 3.0$ \\
\hline
\end{tabular}

\section{Studied mechanical properties:}

Means and standard deviations of compressive strength (CS), elastic modulus $(E)$ and Vickers microhardness (HV) of C-GIC and T-GICs are listed in Table 4. Results of multiple comparisons (Bonferroni test) are shown by superscript letters, $(\mathrm{p} \leq 0.05)$.
Mean data ranged between $(231 \pm 12.39)$ for C-GIC and (212 \pm 7.38$)$ for $10 \%$ T-GIC for CS and between $(15.56 \pm 1.48)$ and $(10.34 \pm 1.47)$ for $E$ and between $(86.87 \pm 4.65)$ and $(79.61(2.29)$ for $\mathrm{HV}$. Addition of $2 \%$ of T1107SB to the GIC powders exhibited a slight change in CS and HV compared to the C-GIC group. The same percent (2\%) showed a more variation in $E$ of the T-GIC groups compared to the control one. Increasing the percent of T1107 SB to GIC powder resulted in a greater variation in the studied mechanical properties with the most increase recorded at $10 \%$.

Statistical analysis revealed significant differences for CS ( $\mathrm{p} \leq 0.05), E(\mathrm{p} \leq 0.001)$ and HV ( $\mathrm{p} \leq 0.001$ ) of studied groups. Though there was a systematic decrease, compared to the control group, in mechanical properties with increasing the 
percent of T1107SB, this decrease was insignificant between C-GIC and those modified with $2 \%$, $4 \%$ and $6 \%$ T1107SB. This applied to the results of CS and HV. Results of $E$, however, revealed insignificant differences between control group and those modified with $2 \%$ and $4 \%$ T1107SB and significant differences upon increasing the percent to $6 \%$ and $10 \%$ in the T-GIC groups.

TABLE (4) Means and standard deviations (in parentheses) of compressive strength, elastic modulus and Vickers microhardness of C-GIC and T-GICs. Each value represents the mean of ten specimens. Different superscript letters indicate statistically significant differences between groups of each column ( $\mathrm{p} \leq 0.05)$.

\begin{tabular}{|c|c|c|c|}
\hline $\begin{array}{c}\text { Properties } \\
\begin{array}{c}\text { Groups } \\
(\text { T1107 \%) }\end{array}\end{array}$ & $\begin{array}{c}\text { Compressive } \\
\text { Strength } \\
(\mathbf{M P a})\end{array}$ & $\begin{array}{c}\text { Elastic Modulus } \\
(\mathbf{G P a})\end{array}$ & $\begin{array}{c}\text { Microhardness } \\
(\text { VHN })\end{array}$ \\
\hline $\begin{array}{c}\text { Group I: } \\
(\mathbf{0} \%)\end{array}$ & $231(12.39)^{\mathrm{a}}$ & $15.56(1.48)^{\mathrm{a}}$ & $86.87(4.65)^{\mathrm{a}}$ \\
\hline $\begin{array}{c}\text { Group II: } \\
(\mathbf{2} \%)\end{array}$ & $230(13.82)^{\mathrm{a}}$ & $14.35(2.11)^{\mathrm{a}, \mathrm{b}}$ & $86.73(3.64)^{\mathrm{a}}$ \\
\hline $\begin{array}{c}\text { Group III: } \\
(\mathbf{4} \%)\end{array}$ & $228(10.47)^{\mathrm{a}}$ & $13.59(1.67)^{\mathrm{a}, \mathrm{b}}$ & $84.51(4.30)^{\mathrm{a}, \mathrm{b}}$ \\
\hline $\begin{array}{c}\text { Group IV: } \\
(\mathbf{6} \%)\end{array}$ & $224(8.35)^{\mathrm{a}, \mathrm{b}}$ & $13.21(1.45)^{\mathrm{b}}$ & $82.72(3.28)^{\mathrm{a}, \mathrm{b}}$ \\
\hline $\begin{array}{c}\text { Group V: } \\
(\mathbf{1 0} \%)\end{array}$ & $212(7.38)^{\mathrm{b}}$ & $10.34(1.47)^{\mathrm{c}}$ & $79.61(2.29)^{\mathrm{b}}$ \\
\hline
\end{tabular}

\section{Fluoride ion release:}

Fluoride ion release (FR) from all groups after immersion in de-ionized water for different times are shown in Table 5. All groups, C-GIC and T-GIC, showed a reduction in FR over the immersion periods selected compared to the starting point ( 24 h). Generally speaking, over the four immersion periods ( $24 \mathrm{~h}, 48 \mathrm{~h}, 1$ week and 1 month), when the added T1107SB was increased in T-GIC groups, a corresponding reduction in FR resulted in compared to C-GIC, with the least at $2 \%$ and the most at $10 \%$.

TABLE (5) Fluoride ion release $(\mu \mathrm{g} / \mathrm{mm} 2)$ from C-GIC and T-GICs after immersion in deionized water for different times.

\begin{tabular}{|c|c|c|c|c|}
\hline \multirow{2}{*}{$\begin{array}{c}\text { Groups (\% of } \\
\text { T1107SB) }\end{array}$} & \multicolumn{4}{|c|}{ Immersion time } \\
\cline { 2 - 5 } & $\mathbf{2 4}$ & $\mathbf{4 8}$ & $\boldsymbol{1}$ & $\boldsymbol{1}$ \\
hours & hours & week & month \\
\hline Group I: (0 \%) & 0.13 & 0.10 & 0.08 & 0.05 \\
\hline Group II: (2 \%) & 0.12 & 0.09 & 0.08 & 0.05 \\
\hline Group III: (4\%) & 0.12 & 0.08 & 0.07 & 0.04 \\
\hline Group IV: (6 \%) & 0.11 & 0.08 & 0.06 & 0.04 \\
\hline Group V: (10 \%) & 0.11 & 0.07 & 0.06 & 0.03 \\
\hline
\end{tabular}

\section{DISCUSSION}

Several studies have been reporting that the growth of a variety of bacterial species was inhibited, both in vitro and in vivo, around conventional and silver GICs, which has generally been attributed to the fluoride released by these materials ${ }^{[30]}$. Opposite to this, there are diverse ideas about the effect of fluoride on secondary caries. Though there are some studies have demonstrated that this problem was reduced with fluoride-containing restorations ${ }^{[31,32]}$, others failed to prove the inhibition effect of fluoride after setting. In addition, other works ${ }^{[33,34]}$ reported no link between fluoride release and bacterial inhibition. Based on this, bacteria molecules may affect the dentin/restoration interface by microleakage causing secondary caries. In the light of this information, various additives were proposed to overcome such shortcomings and to enhance the antimicrobial activities of GICs. 
Improving the antimicrobial activity of these materials to cover a wider range of microorganisms of dental interest is very valuable for both patients and clinicians. In the current study, because of the confirmed antibacterial activity of T1107 Schiff base ${ }^{[23]}$, this material was synthesized and incorporated into the GIC powder to produce a new GIC restorative material with better defense against invading microorganisms.

Choosing the percents of T1107 Schiff base to be added to GIC powders for enhancing their antimicrobial activity was based on a pilot study. Formulating T-GIC groups with just a one-percent difference of added T1107SB showed no or negligible differences between successive groups. Accordingly, a two-percent difference was chosen to formulate our study groups. However, when a statistical analysis was applied to some data, only a significant difference was revealed when the T1107SB percent was increased to $10 \%$. Based on this, four T-GIC groups were formulated in addition to the control group.

With regard to the bacterial species investigated that can be considered as the main limitation of this study, it was really difficult to find all species that are of dental significance such streptococcus mutans which is known as the most cariogenic bacteria that can survive and grow in low-pH environments and Lactobacillus acidophilus which has been found to be the most resistant to the inhibitory effect of GICs ${ }^{[35]}$. Actually, we did our current investigation on Staphylococcus aureus, Bacillus cereus and Candida albicans SC5314 because of their availability at the time of our investigations. In future studies, we may widen the range of bacterial species to be investigated for its sensitivity to these newly modified GICs. Staphylococcus aureus as well as Bacillus cereus are pathogenic bacteria engaged with some oral diseases because of the production of enterotoxins and are of clinical importance. Both types of pathogenic bacteria are Gram-positive.
The antimicrobial activity against these examined strains is strongly dependent on the cationic charge and their chemical (hydrophobic) structures. Cells of Gram-positive bacteria have a simple cell wall structure. The outside cytoplasmic membrane has only a rigid peptidoglycan layer with numerous pores that allow foreign molecules to come into the cell without difficulty. It was believed that the disruption of such microorganisms by polycationic biocides is believed to involve the interaction of the bound polymers with the charged membrane ${ }^{[36]}$. Candida albicans was chosen in this investigation because of its close relationship with some oral diseases as well.

The first null hypothesis was rejected in case of $S$. aureus and B.cereus because of the significant effect of T1107SB in inhibiting their growth compared to C-GIC. Increasing the added percent of T1107SB to GICs produced inhibition zones of wider diameter. This inhibitory effect was more evident with $B$. cereus than $S$. aureus. The antibacterial mechanism of these polymeric materials was thought to be one of these mechanisms: i) adsorption onto the bacterial cell surface, ii) diffusion through the cell wall, iii) binding to the cytoplasmic membrane, iv) disruption of the cytoplasmic membrane, v) precipitation of cell contents and death of the cell and vi) release of Potassium $(\mathrm{K})$ ion and other cytoplasmic constituents ${ }^{[37]}$.

It has been reported that fluoride release of GICs in the immediate vicinity of the cement is the most probable reason for the inhibitory effect on acid production and there is a direct relationship between the fluoride released and the bacterial activity ${ }^{[38,39]}$. Enhanced antimicrobial activity of T-GICs compared to C-GIC can be attributed to one of two mechanisms. The first one is that T1107SB has its own antibacterial action that was added to that of the GIC powder because of fluoride release. The second mechanism is that T1107SB increased the fluoride release to do more inhibition of the bacterial growth. 
The results of the study conducted by Kenawy et al ${ }^{[23]}$ about T1107SB and those of the current study about fluoride release, that was slightly reduced in T-GICs compared to C-GIC, confirm that the first mechanism is more probable.

In case of Candida albicans, however, this hypothesis was accepted because there was no difference between the studied groups. Upon comparing the candida growth inhibition over T-GICs and C-GIC, there was no difference between agar plates. In agreement with our findings, Flisfisch et al. conducted a research to investigate the effect of fluoride on C.albicans. The results showed that there was no effect on their growth where exposure to the fluoride ions was not able to neither alter the cell surface hydrophobicity nor to adhere to the epithelial cells of their membranes ${ }^{[40]}$.

As addition of T1107SB to the GICs demonstrated a significant reduction in $\mathrm{CS}, E$, HV and FR, the second null hypothesis was rejected. Having a deeper insight into the results of CS, E and $\mathrm{HV}$, it can be clearly observed that with only a relatively high percent of $\mathrm{T} 1107 \mathrm{SB}$, there was a decrease in these properties that are of clinical significance. This reduction can be attributed to the polymeric nature of the added T1107SB which is lower than the ceramic glass powder of the GIC in terms of mechanical behavior. With other percents, however, the decrease can be considered minimal and will not deteriorate the material. Though, there was a reduction in FR for all groups, compared to the control group, there will be a compensation from the added T1107SB antibacterial activity when added to the GIC powder.

The added T1107SB is deep red in color, so its discoloration on the white GIC powder was evident. As the modern dental profession is greatly concerned with the aesthetics and tooth-color matching, this modified GIC may not be the best material to be used in aesthetic areas. Instead, this material, with its different percents of added Tetronic material, can be used in posterior fillings of deciduous teeth, as a base under restorations in deep dental cavities, as a core-building material, as a luting cement under crowns that will not reflect the underlying reddish color, ....etc.

\section{CONCLUSIONS}

Within the limitations of this study, it can be concluded that:

- Addition of T1107SB to GIC powder is promising in enhancing the antibacterial activity of GIC materials

- Despite the reduction in CS, E, HV and FR because of the addition of T1107SB, such modified T-GICs can be used with a guaranteed degree of clinical success.

- Opposite to S. aureus and B.Cereus, C.albicans showed no sensitivity to the newly synthesized T-GICs.

\section{REFERENCES}

1. Sajjad A, Wan Bakar WZ, Mohamad D, Kannan TP. Various recent reinforcement phase incorporations and modifications in glass ionomer powder compositions: A comprehensive review. J Int Oral Health 2018; 10:161-167.

2. Sidhu SK, Nicholson JW. A review of glass-ionomer cements for clinical dentistry. J Funct Biomater. 2016; $7: 16-25$.

3. Moshaverinia A, Ansari S, Movasaghi Z, Billington RW, Darr JA, Rehman IU. Modification of conventional glassionomer cements with $\mathrm{N}$-vinylpyrrolidone containing polyacids, nano-hydroxy and fluoroapatite to improve mechanical properties. Dent Mater. 2008; 24: 1381-1390.

4. McCabe J F. Resin-modified glass-ionomers. Biomaterials 1998; 19: 521-527.

5. Cattani-Lorente M A, Dupuis V, Payan J, Moya, F, Meyer J M. Effect of water on the physical properties of resin-modified glass ionomer cements. Dent Mater. 1999; 15: 71-78.

6. Hafshejani TM, Zamanian A, Venugopal JR, Rezvani Z, Sefat F, Saeb MR, et al. Antibacterial glass-ionomer cement restorative materials: A critical review on the 
current status of extended release formulations. J Control Release. 2017; 262:317-328.

7. Loyola-Rodriguez JP, Garcia-Godoy F, Lindquist R. Growth inhibition of glass ionomer cements on mutans streptococci. Pediatr Dent. 1994; 16:346-349.

8. Marti LM, Mata MD, Ferraz-Santos B, Azevedo ER, Giro EMA, Zuanon ACC. Addition of chlorhexidine gluconate to glass ionomer cement: a study on mechanical, physical and antibacterial properties, Braz Dent J, 2014; 25:33-37.

9. Du X, Huang X, Huang C, Frencken J, Yang T. Inhibition of early biofilm formation by glass-ionomer incorporated with chlorhexidine in vivo: a pilot study, Aust Dent J,2012; 57:58-64.

10. Hu J, Du X, Huang C, Fu D, Ouyang X, Wang Y. Antibacterial and physical properties of EGCG-containing glass ionomer cements. J Dent, 2013; 41:927-934.

11. Xie D, Weng Y, Guo X, Zhao J, Gregory RL, Zheng C. Preparation and evaluation of a novel glass-ionomer cement with antibacterial functions. Dent. Mater, 2011; 27:487-496.

12. Weng Y, Howard L, Chong VJ, Sun J, Gregory RL, Xie D. A novel furanone-modified antibacterial dental glass ionomer cement. Acta Biomaterialia, 2012; 8:3153-3160.

13. Hatunoglu E, Öztürk F, Bilenler T, Aksakalli S, Simsek N. Antibacterial and mechanical properties of propolis added to glass ionomer cement. Angle Orthod, 2013; 84:368-373.

14. Mishra A, Pandey RK, Manickam N. Antibacterial effect and physical properties of chitosan and chlorhexidinecetrimide-modified glass ionomer cements. J Indian Soc Pedodont Prev Dent, 2017; 35:28-33.

15. Dhar DN, Taploo CL. Schiff bases and their applications. J Sci Ind Res 1982; 41:501-506.

16. Przybylski P, Huczynski A, Pyta K, Brzezinski B, Bartl F. Biological properties of Schiff bases and azo derivatives of phenols. Curr Org Chem 2009; 13:124-148.

17. da Silva CM, da Silva DL, Modolo LV, Alves RB, de Resende MA, Martins CVB, de Fatima A. Schiff bases: A short review of their antimicrobial activities. J Adv Res, 2011; 2:1-8

18. de Souza AO, Galetti FCS, Silva CL, Bicalho B, Parma MM, Fonseca SF. Antimycobacterial and cytotoxicity activity of synthetic and natural compounds. Quimica Nova Journal, 2007; 30:1563-1566.
19. Guo Z, Xing R, Liu S, Zhong Z, Ji X, Wang L, et al. Antifungal properties of Schiff bases of chitosan, N -substituted chitosan and quaternized chitosan. Carbohydr Res, 2007; 342:1329-1332.

20. Mansur CR, Barboza SP, González G, Lucas EF. PLURONIC $\times$ TETRONIC polyols: study of their properties and performance in the destabilization of emulsions formed in the petroleum industry. J Colloid Interface Sci, 2004; 271:232-240.

21. Rey-Rico A, Cucchiarini M. PEO-PPO-PEO Tri-Block Copolymers for Gene Delivery Applications in Human Regenerative Medicine-An Overview. Int J Mol Sci, 2018;19:775.

22. Chiappetta DA, Alvarez-Lorenzo C, Rey-Rico A, Taboada P, Concheiro A, Sosnik A. N- alkylation of poloxamines modulates micellar assembly and encapsulation and release of the antiretroviral efavirenz. Eur J Pharm Biopharm, 2010; 76:24-37.

23. Kenawy E-R, Azaam MM, Khattab SA. Synthesis and Antibacterial Activity of Schiff base Compounds Based on Poloxamine. Scientific Journal of Basic and Applied Sciences, 2019; 40:69-77.

24. Denitsa, N., and Mariana, N. (2005). Screening the antimicrobial activity of Actinomycetes strains isolated from Antarctica. J Cul Coll, 2004-2005; 4: 29-35.

25. International Organization for Standardization. DentistryWater-based cements Part 1: Powder/liquid acid-base cements 2007.

26. Yli-Urpo H, Lassila LVJ, Narhi T, Vallittu PK. Compressive strength and surface characterization of glass ionomer cements modified by particles of bioactive glass. Dent Mater 2005; 21:201-209.

27. Moshaverinia A, Brantley WA, Chee WW, Rohpour N, Ansari S, Zheng F. Measure of microhardness, fracture toughness and flexural strength of N-vinylcaprolactam (NVC)-containing glass ionomer dental cements. Dent Mater 2010; 26:1137-1143.

28. Moshaverinia M, Navas A, Jahedmanesh N, Shah KC, Moshaverinia A, Ansari S. Comparative evaluation of the physical properties of a reinforced glass ionomer dental restorative material. J Prosthet Dent 2019; 122:154-159.

29. Alatawi RAS, Elsayed NH, Mohamed WS. Influence of hydroxyapatite nanoparticles on the properties of glass ionomer cement. J Mater Res Tech 2019; 8:344-349. 
30. Yadiki JV, Jampanapalli SR, Konda S, Inguva HC, Chimata VK. Comparative Evaluation of the Antimicrobial Properties of Glass Ionomer Cements with and without Chlorhexidine Gluconate. Int J Clin Pediatr Dent 2016; 9:99-103.

31. Hicks MJ, Flaitz CM. Resin-modified glass-ionomer restorations and in vitro secondary caries formation in coronal enamel, Quintessence Int. (Berlin, Germany: 1985) 31 (8) (2000) 570-578.

32. Savarino L, Breschi L, Tedaldi M, Ciapetti G, Tarabusi C, M. Greco M. Ability of restorative and fluoride releasing materials to prevent marginal dentine demineralization, Biomaterials 25 (6) (2004) 1011-1017.

33. Forss H, Widström E. Reasons for restorative therapy and the longevity of restorations in adults, Acta Odontol. 62 (2) (2004) 82-86.

34. Manhart J, Garcia-Godoy F, Hickel R. Direct posterior restorations: clinical results and new developments. Dent. Clin. N. Am. 46 (2) (2002) 303-339.

35. Mittal S, Soni H, Sharma DK, Mittal K, Pathania V, Sharma S. Comparative evaluation of the antibacterial and physical properties of conventional glass ionomer cement containing chlorhexidine and antibiotics. J Int Soc Prev Community Dent. 2015;5(4): 268-275.

36. Kanazawa TA, Ikeda T, Endo T. Antibacterial activity of polymeric sulfonium salts. J Polym Sci Part A: Polym. Chem. 1993: 31, 3003.

37. Kawabata N, Nishiguchi M. Antimicrobial Materials for Biomedical Applications. Environ. Microbiol. 1988, 54: 2532-2539.

38. Shashibhushan KK, Basappa N, Subba Reddy VV. Comparison of antibacterial activity of three fluorides and zinc releasing commercial glass ionomer cements on strains of mutans streptococci: an in vitro study. J Indian Society of Pedodontics and Preventive Dentistry 2008; 26: 56-S61.

39. Vermeersch G, Leloup G, Delmee M, Vreven J. Antibacterial activity of glass-ionomers, compomers and resin composites: relationship between acidity and material setting phase. J Oral Rehabil 2005 May; 32:368-374.

40. Flisfisch S, J Meyer J, Meurman JH, Waltimo T. Effects of fluorides on Candida albicans. Oral Diseases 2008; 14 , 296-301. 